\title{
Beam complexity and monitor unit efficiency comparison in two different volumetric modulated arc therapy delivery using automated planning
}

\section{Chengqiang $\mathrm{Li}$}

Shandong Cancer Hospital and Institute, Shandong First Medical University and Shandong Academy of Medical Sciences https://orcid.org/0000-0002-8549-2416

\section{Cheng Tao}

Shandong Cancer Hospital and Institute

\section{Tong Bai}

Shandong Cancer Hospital and Insitute

\section{Zhenjiang Li}

Shandong Cancer Hospital and Institute

\section{Ying Tong}

Shandong Cancer Hospital and Institute

Jian Zhu

Shandong Cancer Hospital and Institute

\section{Yong Yin}

Shandong Cancer Hospital and Institute

Jie Lu ( $\square$ lljie1987@126.com )

Department of Radiation Oncology Physics, Shandong Cancer Hospital and Institute, Shandong First Medical University and Shandong Academy of Medical Sciences https://orcid.org/0000-0002-87898129

\section{Research article}

Keywords: Volumetric modulated arc therapy, Beam complexity, Monitor unit efficiency, Auto-Planning, Breast cancer, Nasopharyngeal carcinoma

Posted Date: August 26th, 2020

DOI: https://doi.org/10.21203/rs.3.rs-23401/v2

License: (c) (i) This work is licensed under a Creative Commons Attribution 4.0 International License.

Read Full License 
Version of Record: A version of this preprint was published on March 10th, 2021. See the published version at https://doi.org/10.1186/s12885-021-07991-6. 


\section{Beam complexity and monitor unit efficiency comparison in two different}

volumetric modulated arc therapy delivery using automated planning Chengqiang Li, Cheng Tao, Tong Bai, Zhenjiang Li, Ying Tong, Jian Zhu, Yong Yin $^{*}$, Jie $L u^{*}$

Department of Radiation Oncology Physics, Shandong Cancer Hospital and Institute, Shandong First Medical University and Shandong Academy of Medical Sciences, Jinan 250117, China Corresponding author: Yong Yin, Jie Lu Email: yinyongsd@126.com, Iljie1987@126.com

\section{Abstract}

Background: To investigate the beam complexity and monitor unit (MU) efficiency issues for two different volumetric modulated arc therapy (VMAT) delivery technologies for patients with left-sided breast cancer (BC) and nasopharyngeal carcinoma (NPC).

Methods: Twelve left-sided BC and seven NPC cases were enrolled in this study. Each delivered treatment plan was optimized in Pinnacle ${ }^{3}$ treatment planning system with Auto-Planning module for Trilogy and Synergy systems. Similar planning dose objectives and beam configuration were used for each site in two different delivery systems to produce clinically acceptable plans. Beam complexity was evaluated in terms of segment area(SA), segment width(SW), leaf sequence variability(LSV), aperture area variability(AAV), modulation complexity score(MCS) based on MLC sequence and MU. Plan delivery and gamma evaluation were performed using helical diode array. Results: With similar plan quality, the average SAs for the Trilogy plans were smaller than those for the Synergy plans: $55.5 \pm 21.3 \mathrm{~cm}^{2}$ vs. $66.3 \pm 17.9 \mathrm{~cm}^{2}$ $(p<0.05)$ for the NPC cases, and $100.7 \pm 49.2 \mathrm{~cm}^{2}$ vs. $108.5 \pm 42.7 \mathrm{~cm}$ ${ }^{2}(p<0.05)$ for BC cases, respectively. The SW was statistically significant for two delivery systems (NPC: $6.87 \pm 1.95 \mathrm{~cm}$ vs.6.72 $\pm 2.71 \mathrm{~cm}, \mathrm{p}<0.05$; $\mathrm{BC}$ : $8.84 \pm 2.56 \mathrm{~cm}$ vs. $8.09 \pm 2.63 \mathrm{~cm}, \mathrm{p}<0.05)$. LSV was statistically significant smaller for Trilogy (NPC: $0.84 \pm 0.033$ vs. $0.86 \pm 0.033, p<0.05$; BC: $0.89 \pm 0.026$ vs.0.90 $\pm 0.26, p<0.05)$. The mean AAV was statistically significant larger for the Trilogy than Synergy (NPC: $0.18 \pm 0.064$ vs.0.14 $\pm 0.037, p<0.05$; BC: $0.46 \pm 0.15$ vs. $0.33 \pm 0.13, p<0.05)$. The MCS values for the Trilogy were higher than those for the Synergy: $0.14 \pm 0.016 \mathrm{vs} .0 .12 \pm 0.017(p<0.05)$ for the NPC cases, and $0.42 \pm 0.106$ vs. $0.30 \pm 0.087(p<0.05)$ for the $B C$ cases. Compared with Synergy plans, the average MU for Trilogy plans were larger: 828.6 $\pm 74.1 \mathrm{MU}$ and $782.9 \pm 85.2 \mathrm{MU}(\mathrm{p}>0.05)$ for the NPC cases, and 444.8 $\pm 61.3 \mathrm{MU}$ and 393.8 $\pm 75.3 \mathrm{MU}(\mathrm{p}>0.05)$ for the BC cases. The gamma index agreement scores were never below $91 \%$ using $3 \mathrm{~mm} / 3 \%$ (global) distance to agreement and dose difference criteria and a $10 \%$ lower dose exclusion threshold.

Conclusions: The pinnacle ${ }^{3}$ Auto planning system can optimize BC and NPC plans to obtain the same plan quality using Trilogy and Synergy systems. We 
found that this two systems resulted in different SA, SW, LSV, AAV and MCS. As a result, we suggested that beam complexity should be considered in providing further methodologies while optimizing VMAT auto planning.

Keywords: Volumetric modulated arc therapy, Beam complexity, Monitor unit efficiency, Auto-Planning, Breast cancer, Nasopharyngeal carcinoma

\section{Background}

Currently, volumetric modulated arc therapy (VMAT) is one of the most advanced delivery techniques in radiotherapy, which simultaneously integrates multi-leaf collimator (MLC) field shape modulation with gantry speed and dose rate variations. With more degrees of freedom during treatment, advanced arc plans provide a larger flexibility in shaping dose distributions and delivery the plan more efficiently than other static beams plans [1,2]. VMAT technique has become clinically and commercially available by using Trilogy (Varian Medical Systems, Palo Alto, CA, USA) and Synergy (Elekta AB, Stockholm, Sweden) linear accelerators. However, there are many differences between the two machines, such as the method of dose rate control [3], MLC leaf width [4]and the jaw tracking technique [5]. Several investigations have been performed in order to show dosimetric effect on these system differences.

Studies have suggested that VMAT may be useful in a variety of treatment sites, such as BC [6-8] and NPC [9-12]. As well all known that high quality treatment plan relies on the skills and experience of the dosimetrist, which can vary greatly. Recently, some automatic planning solutions have been developed, such as atlas-based planning, ideal dose distribution estimation and template-based optimization [13].

The template-based Auto-Planning module has recently become clinically available in Pinnacle ${ }^{3}$ radiation therapy treatment planning system (TPS). Several comprehensive studies have been performed to evaluate the benefit of this new auto-planning system [13-16]. The results showed that the Auto-Planning VMAT technique achieved adequate target dose coverage while maintaining low doses to organs-at-risk, and therefore reduced the potential for induction of second malignancy and side effects. Another conclusion from existing research results was that the auto-planning module could generate plans with consistent quality.

Due to the highly choreographed nature of VMAT delivery, many potential sources of error arise, necessitating patient specific quality assurance (QA) and dosimetric verification technique [17]. The treatment plan of VMAT requires compromise between dose conformity (complexity) and deliverability. The creation of modulation complexity score (MCS) [18] based on plan parameters allows for a quantitative assessment of plan complexity and can provide more information related to dose delivery than simple beam parameters such as monitor units (MU). Excessive complexity in VMAT plans increases the dose uncertainty, prolongs the treatment time, and increases the 
susceptibility to changes in patient or target geometry. McNiven et al. [19] performed a study to evaluate the utility of MCS to evaluate the relationship of the metric with deliverability in IMRT and reported that different clinical treatment sites have an inherent difference in the level of complexity, the average MCS of IMRT plans for head and neck as well as breast is 0.165 and 0.909 , respectively.

However, the use of MCS for auto VMAT plan for different treatment sites is still rare. The purpose of this study was to evaluate beam complexity of two delivery systems optimized by the Auto-Planning.

\section{Methods}

\section{Patients}

A retrospective analysis was performed on 12 patients with left-sided $B C$ and 7 patients with NPC who were randomly selected from our institution. The prescription dose was 50.0Gy in 25 fractions for BC. There were three different dose levels in NPC patients included 54 Gy, 60 Gy, and 66 Gy in 30 treatment fractions with a simultaneous integrated boost technique.

For BC, plan objectives were: D100\% $>100 \%$ for CTV, D100\% >90\% for PTV, mean dose for heart and contralateral breast. $V_{x G y}$ to ipsilateral lung and body were minimized to keep the dose to the organ at risks (OARs) as low as possible by setting higher priority upon avoidance of the contralateral breast, lungs and heart, without compromising the PTV dose coverage and maximum hotspots should not exceed $110 \%$.

For NPC, the treatment goals were that $95 \%$ of PTV should receive more than $95 \%$ of the prescribed dose and the maximum dose should below $110 \%$ of prescription dose. Regarding to the OARs, the maximum doses to the brain stem and the spinal cord were set to 54Gy and 45Gy, respectively. In addition, at least one side of the parotid glands should receive a mean dose less than $26 \mathrm{~Gy}$, or the volume receiving $30 \mathrm{~Gy}$ radiation should be $<50 \%$. The dose to other normal tissues was minimized within a reasonable range without affecting the target coverage.

\section{Treatment optimization}

The treatment planning was performed with Pinnacle $^{3}$ (V9.10, Philips Radiation Oncology Systems, Fitchburg, WI) treatment planning system. Each of the 19 treatment plans was optimized with Pinnacle Auto-Planning module for a Varian Trilogy (Varian Medical Systems, Palo Alto, CA) linear accelerator, equipped with a 120 leaf Millennium MLC and an Elekta Synergy (Elekta Ltd, Crawley, UK) linear accelerator, equipped with a 40 leaf MLCi using $6 \mathrm{MV}$ photons, respectively. Varian Trilogy system can delivery VMAT plan using continuously variable dose rate while Elekta Synergy using binned variable dose rate. Trilogy system was equipped with a 120 Millennium MLC, the Millennium 120 multi-leaf collimators (MLCs) consists of two banks of 60 MLC leaves, with the outer 20 and inner 40 on each side having widths of $10 \mathrm{~mm}$ 
and $5 \mathrm{~mm}$, respectively. Synergy system was equipped with MLCs comprising 40 leaf pairs projected leaf width of $10 \mathrm{~mm}$ at the isocenter. In addition, Synergy provides jaw tracking technique, Trilogy doesn't have jaw tracking capability.

The NPC VMAT plans in this study used two full arcs with gantry angles $181^{\circ}-179^{\circ}$ for a CW rotation and $179^{\circ}-181^{\circ}$ for a CCW rotation, collimator angles were set to be $15^{\circ}$ and $345^{\circ}$ to avoid possible overlapping tongue and groove effect. The BC VMAT plans consisted of two short partial arcs, each arc consisted of a $40^{\circ}$ gantry rotation, irradiating between gantry angles $296^{\circ}-336^{\circ}$ and $104^{\circ}-144^{\circ}$ for a CW rotation with collimator angles of $5^{\circ}-10^{\circ}$ for decreasing the larger volume of low dose spread to healthy tissue based on our experience. The dose calculations were performed using Pinnacle's collapsed cone convolution superposition algorithm with the gantry spacing resolution $2^{\circ}$ and the dose voxel size of $0.3 \times 0.3 \times 0.3 \mathrm{~cm}^{3}$.

The Auto-Planning module requires the user to define a template with prioritized optimization goals for PTV coverage and dose limits for OARs. These prioritized optimization goals are used by the Auto-planning engine to formulate optimization objectives. Multiple optimization loops iteratively reformulate and adjust the optimization objectives to meet the criteria defined in the template. During the optimization process, the optimization of target coverage has higher priority over dose reduction to OARs. After the auto-optimization, the treatment plans were fine-tuned by an expert planner to adapt the PTV coverage and OARs protection as same as possible by the two delivery systems.

\section{Complexity scores}

To optimize arc delivery treatment plans, the treatment planning system uses multiple control points (CP) to represent the arc plan. The CP refers to the instantaneous configuration at a point in time and 'segment' refers to the duration between CPs, although the two may be used synonymously. Once treatment plan is completed for a patient, the plans were extracted from the treatment planning system as RTPlan files. RTPlan files contain the information of gantry angle, MLC configuration, Jaw position, and MU for each CP. As main part of our work, the beam complexity scores and dose-volumetric parameters were calculated by an in-house software developed in Matlab (Version 2010b, MathWorks, Natick, MA).

The complexity scores include MU, segment width (SW), segment area(SA), leaf sequence variability(LSV), aperture area variability (AAV) and modulation complexity score(MCS). Based on Mcniven et al. [19] and Rajasekaran et al. [20] formalism, the beam complexity scores above and dose-volumetric parameters were calculated by an in-house software developed in Matlab (Version 2010b, MathWorks, Natick, MA). The SW and SA for a given segment at a certain gantry angle is as described by Eq. (1) and (2), respectively, where pos is the coordinate of the leaf position and $\mathrm{N}$ is the number of in-field moving leaves inside the jaw position. $\mathrm{N}$ is the total number 
of leaf pairs, $n$ is the index number of leaf pairs. LeafWidth $n$ is the width of the nth leaf pair. $\mathrm{SW}_{\text {segment }_{\mathrm{i}}}$ can be roughly understood as the area of a beam segment. The leaves that remained closed during treatment were not considered.

$\mathrm{SW}_{\text {segment }_{\mathrm{i}}}=\max \left(\operatorname{pos}_{\mathrm{n} \in \mathrm{N}_{\text {left bank }}}-\operatorname{pos}_{\mathrm{n} \in \mathrm{N}_{\text {right bank }}}\right)$,

$\mathrm{SA}_{\text {segment }_{\mathrm{i}}}=\sum_{\mathrm{n}=1}^{\mathrm{N}}\left(\left\langle\operatorname{pos}_{\mathrm{n}}\right\rangle_{\text {left bank }}-\left\langle\operatorname{pos}_{\mathrm{n}}\right\rangle_{\text {right bank }}\right) \times$ LeafWidth $_{\mathrm{n}}$,

(2)

MCS and its parameters for each VMAT plans were determined by following formulae:

$\operatorname{pos}_{\text {max }}\left(\operatorname{segment}_{\mathrm{i}}\right)=\left\langle\max \left(\operatorname{pos}_{\mathrm{n} \in \mathrm{N}}\right)-\min \left(\operatorname{pos}_{\mathrm{n} \in \mathrm{N}}\right)\right\rangle_{\mathrm{MLC} \text { bank }}$,

$\mathrm{LSV}_{\text {segment }_{\mathrm{i}}}=\left\langle\frac{\sum_{\mathrm{n}=1}^{\mathrm{N}-1}\left(\operatorname{pos}_{\max }-\left|\left(\operatorname{pos}_{\mathrm{n}}-\operatorname{pos}_{\mathrm{n}+1}\right)\right|\right)}{(\mathrm{N}-1) \times \operatorname{pos}_{\max }}\right\rangle_{\text {left bank }} \times\left\langle\frac{\sum_{\mathrm{n}=1}^{\mathrm{N}-1}\left(\operatorname{pos}_{\max }-\left|\left(\operatorname{pos}_{\mathrm{n}}-\operatorname{pos}_{\mathrm{n}+1}\right)\right|\right)}{(\mathrm{N}-1) \times \operatorname{pos}_{\max }}\right\rangle_{\text {right bank }}$,

$\mathrm{AAV}_{\text {segment }_{i}}=\frac{\sum_{\mathrm{n}=1}^{\mathrm{N}}\left(\left\langle\operatorname{pos}_{\mathrm{n}}\right\rangle_{\text {left bank }}-\left\langle\operatorname{pos}_{\mathrm{n}}\right\rangle_{\text {right bank }}\right) \times \text { LeafWidth }_{\mathrm{n}}}{\sum_{\mathrm{n}=1}^{\mathrm{N}}\left(\left\langle\operatorname{pos}_{\mathrm{n}}\right\rangle_{\text {left bankearc }}-\left\langle\operatorname{pos}_{\mathrm{n}}\right\rangle_{\text {right bank } \in \text { arc }}\right) \times \text { LeafWidth }_{\mathrm{n}}}$,

(5)

The parameter leaf sequence variability (LSV) was used to characterize the variation in segment shape and parameter aperture variability (AAV) was used to characterize the variation in segment area relative to the maximum aperture defined by all the segments. For a given arc of many small segments that are spatially separated from each other, the values of LSV and AAV decrease.

To summarize the influence of LSV, AAV and MU, the MCS for a given arc is calculated based on the scores above, as Eq. (6) describes:

$\mathrm{MCS}_{\text {arc }}=\sum_{\mathrm{i}=1}^{\mathrm{I}-1}\left[\frac{\mathrm{LSV}_{\text {segment }_{\mathrm{i}}}+\mathrm{LSV}_{\text {segment }_{\mathrm{i}+1}}}{2} \times \frac{\mathrm{AAV}_{\text {segment }_{\mathrm{i}}}+\mathrm{AAV}_{\text {segment }_{\mathrm{i}+1}}}{2} \times\right.$ weight $\left._{\mathrm{i}}\right]$.

(6)

The MCS calculation based on three parameters: segment shape, area and weight, as in the original definition, has the value range from 0 to1. MCS $=1$ means no modulation and these interpretations suggest that the average MCS score for a treatment site always decreases with increased inherent complexity.

\section{Dosimetric QA, gantry speed and dose rate comparisons}

For dosimetric QA, the VMAT plans were delivered while irradiating a helical diode array dosimeter (ArcCHECK, Sun Nuclear corporation). The measurements were compared to TPS predictions using SNC patent software with the evaluation criteria $3 \mathrm{~mm}$ distance to agreement and $3 \%$ (global) dose difference $(3 \mathrm{~mm} / 3 \%), 2 \mathrm{~mm} / 3 \%$ and $2 \mathrm{~mm} / 2 \%$ with a lower dose exclusion threshold of $10 \%$. Virtual inclinometer was utilized to record the time when gantry rotated to the angle specified by each control point and the time between control points. The gantry speed and dose rate were calculated using 
the RTPlan files, acm files and virtual inclinometer as Wang et al. reported [21].

\section{Evaluation}

The collected data represented as mean \pm standard deviation was analyzed with SPSS software (version 13.0, Chicago, USA). The difference of Trilogy and Synergy plans were evaluated by the two-sided Wilcoxon matched-pair signed-rank test. The threshold for statistical significance was set at $p<0.05$.

\section{Results}

\section{PTVs coverage and OARs sparing}

Average DVHs for the target volumes and the various OARs comparison between Trilogy and Synergy plans for NPC and BC were shown in Figure 1. All plans sufficiently respected the planning objectives and could be clinically accepted. After post-optimization sequenced by Auto-Planning, there were no drastic variation in the dose-volume between PTVs and OARs.

\section{MU efficiency}

In Table 1, the average volume of PTV and number of MUs are summarized. The average MU among BC patients was $444.8 \pm 61.3$ for Trilogy and $393.8 \pm 75.3$ for Synergy. The average MU among NPC patients was $828.6 \pm 74.1$ for Trilogy and $782.9 \pm 85.2$ for Synergy.

\section{Complexity scores comparison}

MCS and its dependent parameters such as SA, LSV and AAV are summarized in Table 2. Significant changes were observed between the Trilogy and Synergy plans in terms of beam complexity scores for both NPC and BC plans $(p<0.05$ for all). The detailed SA and AAV comparisons of the Trilogy and the Synergy plans for each control point are given in Figures 2 and 3.

\section{Dosimetric QA, gantry speed and dose rate comparisons}

Table 3 presented a summary of gantry speed, dose rate and volumetric gamma evaluation $(3 \mathrm{~mm} / 3 \%, 2 \mathrm{~mm} / 3 \%$ and $2 \mathrm{~mm} / 2 \%$ ) results of ArcCHECK. For both Trilogy and Synergy, the gantry speed of NPC plans were greater than $4 \%$ s. The mean gantry speed of Breast plans were $2.6 \%$ and $1.1 \%$ s for Trilogy and Synergy, respectively. For NPC plans, the average passing rates $(3 \mathrm{~mm} / 3 \%)$ of Trilogy and Synergy were $95.2 \%$ and $93.3 \%$, respectively. For Breast plans, the average passing rates $(3 \mathrm{~mm} / 3 \%)$ of Trilogy and Synergy were $94.5 \%$ and $93.7 \%$, respectively.

\section{Discussion}

This work demonstrated that the Pinnacle ${ }^{3}$ Auto-Planning system was able to produce comparable quality VMAT plans by different delivery systems, and 
was therefore able to successfully handle the geometric and dosimetric variations between NPC and BC sites. The significant differences in modulation complexity of VMAT plans between Trilogy and Synergy were found.

The dosimetric studies of auto plan showed excellent target volume coverage and OARs sparing to various target paradigms [13-16]. Hazell et al. [14] compared auto plans with manual head and neck cancer IMRT treatment plans, they found that target coverages in the auto plans were similar to manual plans, but automatically generated plans had less irradiation of healthy tissue. In contrast to other studies, the primary goal in this study was to generate "same" plans.

There were several major differences between Varian Trilogy and Elekta Synergy systems used for delivering VMAT plans. To reduce the variability in the quality of treatment plans, dose objectives in Auto-Planning can be automatically generated to drive the optimization of the new plan. What is noteworthy is that it required further user intervention to manually set optimization objectives at the end of the auto optimization process. Allowing for a manual post optimization, the coverage for PTVs and dose constrains for OARs were as same as possible.

In summary, as shown in Figure1, the Pinnacle Auto-Planning was able to produce VMAT comparable plans using Trilogy and Synergy delivery systems for more complex cases (breast and NPC tumor regions). For NPC plans, the differences occurred about brainstem and spinal cord because the concern was mostly focused to constraint the max dose of these two organs during optimization. However, the values were still within the tolerance range. In this study, MLC width and the jaw tracking capability were the major parameters influencing the dose distribution. Intuitively, a finer leaf width should result in more conformal target shaping. Lafond et al. [22] showed that Beam Modulator (4mm leaf width) and MLCi2 (10 mm leaf width) MLCs from Elekta provided satisfactory dose distributions for head and neck cancer VMAT. OAR sparing was better for brainstem and spinal cord in Beam Modulator. However, delivery efficiency of VMAT plans was better than MLCi2 with lower MU. Our results are in agreement with their study. It is possible that the jaw tracking and lager segment area mainly decreases the MLC transmission which was the likely explanation to decreased low dose. The reduction of low dose irradiation of lungs and lens was found in Synergy plans in this study. Due to the limited number of cases and the specific site studied, we do not intend the results to be generalizable. A study by Height et al. [23] investigated the effect of different leaf widths from Varian on the treatment of early breast treatment and found that no clinically significant differences were found using $5 \mathrm{~mm}$ vs. 10 $\mathrm{mm}$ MLC leaf widths. A previous study by French et al. [24] investigated transferring high-definition MLC (14-32-14 pattern of widths 5-2.5-5 mm) VMAT plan to Millennium MLCs (10-40-10 pattern of widths $10-5-10 \mathrm{~mm}$ ) and found that high-definition MLC had smaller area for a given control point. In 
addition to differences in leaf numbers and widths, Trilogy and synergy differ in jaw movement and dose rate bins. Nevertheless, the plan qualities appeared to be equivalent if considered from a clinical perspective for both delivery techniques.

Regarding the treatment MU, the corresponding data listed in Table 1 suggested that although the tumor volumes were similar for NPC and BC, the BC plans had lower MUs than NPC plans by applying approximately tangential arcs. The Synergy required less MU to treat the same volume tumor, even there was not drastic variation in the MU value among the two systems for either site. This difference of MU was largely attributed to the fact that large area segments were more often in Synergy plans than in Trilogy plans. Similar study in terms of IMRT plans was reported by Qi et al. [25]. As observed from their published study, high MU efficiency was observed in direct aperture optimization (DAO) plans than direct machine parameter optimization (DMPO) plans because large area segments were used often in DAO plans.

Both Synergy and Trilogy plans had clinically acceptable plan quality, but we observed that the SA was different. The breast plans used larger SA than NPC plans. Synergy used larger SA than Trilogy for the same site. The Synergy plans were manifested in the larger segment area and lower MU relative to the Trilogy plans. Furthermore, VMAT auto plans were more sensitive to MLC errors [26].

To assess the beam complexity, as stated in the results, we found significant differences. In this study, the MCS of NPC and BC plans were 0.14 and 0.42 for Trilogy, 0.12 and 0.3 for Synergy. Dhanabalan et al. [20] reported that average MCS for the head and neck VMAT plans were 0.2224 . The MCS for auto plans were smaller than manual plans, which suggested the beams were more complex. The autoplans were more modulated as illustrated by Hansen et al. [13], which might cause the slightly lower pass rate of $97.7 \%$ in the ArcCHECK measurements. The question of the relationship of plan complexity and gamma index analysis of delivery accuracy was not included in this study. Regarding gantry rotation speed variations, both Trilogy and Synergy can rotate at high gantry speed when delivering the full-arc NPC plans. However, for the limited angle Breast VMAT plans, the gantry speed of the Trilogy and Synergy was different. As shown in Table 3, the Trilogy gantry speed is half that of NPC with higher dose rate while Synergy reduced the gantry speed to $1 / 4$ of NPC for Breast. Again, it was possible to deliver a VMAT plan faster with Trilogy than with Synergy, which was comparable to values reported in other studies with the finding of Osborn et al. [27] It is noteworthy that the gantry speed and dose rate were calculated from one control point to next control point. Virtual inclinometer for recording gantry angle is an effective way to record the time between control points that has demonstrated accurate and high reproducibility [21]. 
However, much effort has been devoted to analysis that correlation using different patient-specific QA phantom. Crowe et al. [28] found that the 'small aperture score' provided threshold values which successfully distinguished deliverable treatment plans from plans that did not pass QA using MapCheck2 diode array. Li et al. [29] found that the planning parameters such as the average leaf travel and average field indicating a correlation between the plan complexity and the passing rate using ArcCHECK diode array. Dhanabalan et al. [20] studied the correlation results between the MCS and gamma analysis results indicate that MCS of a plan has a weak correlation with the planar as well as volumetric gamma analysis passing rates using Octavius4D phantom. Knowledge of this relationship will be further accumulated using our MatrixX and ArcCHECK phantom. This study demonstrates that treatment techniques differ in terms of treatment MU and MCS. Mcgarry et al. [30] suggested MCS was most suitable for inclusion within the cost function to limit complexity in IMRT optimization. VMAT plans, which are less complex, also have higher probabilities of yielding accurate dosimetric results. Further investigations are in progress to confirm the relationship between complexity scores and delivery prediction errors. In generally, current TPSs use optimization algorithm to balance competing dose volume objectives and ignore the complexity. In our viewpoint, when plans are comparable in terms of doses to OARs and PTV coverage, the less complex plan is preferred before it is sent for QA. When 2 plans are comparable in plan qualities using two machines, plan which has higher prediction gamma rate is preferred. When the plans were complex or with lower prediction gamma rate, increasing the number of ARCs or CPs can be used to decrease plan complexity by increasing the number degree of freedom. From the planner's point of view, the plan complex parameters as a part of cost function can be used to guide the plan parameter setting and optimization in TPS. Finally, the monitoring of complexity allows better consistency in the treatment planning progress.

\section{Conclusions}

In conclusion, all VMAT plans using Trilogy or Synergy implemented in the Pinnacle Auto-Planning module were clinically acceptable and comparable in terms of PTV coverage and OAR sparing for NPC and BC. The differences in SA, SW, LSV, AAV and MCS which should be considered in auto-plan designing were observed. In reality, this can be implemented as a function in TPS in the future.

\section{Declarations}

\section{Abbreviations}

VMAT: Volumetric modulated arc therapy; MU: Monitor unit; BC: Breast cancer; NPC: Nasopharyngeal carcinoma; SA: Segment area; SW: segment width; LSV: Leaf sequence variability; AAV: Aperture area variability; MCS: Modulation 
complexity score; MLC: Multi-leaf collimator; TPS: Treatment planning system; QA: Quality assurance; DMPO: Machine parameter optimization; DAO: Direct aperture optimization; OARs: organ at risks;

\section{Ethics approval and consent to participate}

This study was approved by the local ethics committee of Shandong Cancer Hospital and Institute, and each patient gave written and informed consent before the study.

\section{Consent for publication}

Not applicable.

\section{Availability of data and material}

All data generated or analyzed during this study are included in this published article.

\section{Competing interests}

The authors declare that they have no competing interests.

\section{Funding}

This study was partially funded by Natural Science Foundation of China (81671785), Natural Science Foundation of Shandong Province (ZR2016HQ09, ZR2019PH108), Academic promotion program of Shandong First Medical University and the Foundation of Taishan Scholars (No.tsqn201909140), Science and Technology Plan of Jinan city (201907108).

\section{Acknowledgements}

The authors wish to thank patients and their kin for supporting our work and thank editors as well as reviewers for reading the manuscript.

\section{Authors' contributions}

CL conceived the idea and JZ, JL and YY designed the study. CL, CT and TB collected the data. CL, ZL, YT and CT performed the analysis. CL drafted the manuscript with the help of JZ, YY and JL. All authors read and approved the final manuscript.

\section{References}

1. Otto K. Volumetric modulated arc therapy: IMRT in a single gantry arc. Med Phys. 2008; 35: 310-317.

2. Bedford JL. Treatment planning for volumetric modulated arc therapy. Med Phys. 2009; 36: 5128-38.

3. $\mathrm{Y}$ Wu, N Dogan, $\mathrm{X}$ Liang. Assessment of volumetric arc therapy plans for constant and variable dose rates. Med Phys. 2010; 37: 3350-3351

4. Kim J I, Park J M, Park S Y, Choi C H, Wu H G, Ye S J. Assessment of potential jaw-tracking advantage using control point sequences of VMAT planning. J Appl Clin Med Phys. 2014; 15:4625-4628.

5. Serna A, Puchades V, Mata F, Ramos D, Alcaraz M. Influence of multi-leaf collimator leaf width in radiosurgery via volumetric modulated arc therapy and 3D dynamic conformal arc therapy. Phys Med. 2015; 33:293-296.

6. Jeulink M, Dahele M, Meijnen P, Slotman BJ, Verbakel W-FAR. Is there a preferred IMRT technique for left-breast irradiation?. J Appl Clin Med Phys. 2015; 16:5266-74

7. Tsai P F, Lin S M, Lee S H, Yeh C Y, Huang Y T, Lee C C, et al. The feasibility study of using multiple partial volumetric-modulated arcs therapy in early stage left-sided breast cancer patients. J Appl Clin Med Phys. 2012; 13: 3806-3817.

8. Popescu C C, Olivotto I A, Beckham W A, Ansbacher W, Zavgorodni S, Shaffer R, et al. Volumetric modulated arc therapy improves dosimetry and reduces treatment time compared to conventional intensity-modulated radiotherapy for locoregional radiotherapy of left-sided breast cancer and internal mammary nodes. Int J of Radiat Oncol Biol Phys. 2010; 76: 287-295.

9. Lu S H, Cheng J C H, Kuo S H, Lee J-JS, Chen LH, Wu JK, et al. Volumetric modulated arc therapy for nasopharyngeal carcinoma: a dosimetric comparison with TomoTherapy and step-and-shoot IMRT. Radiother Oncol. 2012;104(3): 324-330. 
10. Bertelsen A, Hansen C R, Johansen J, Brink C. Single arc volumetric modulated arc therapy of head and neck cancer. Radiother Oncol, 2010; 95: 142-148.

11. Ning Z H, Mu J M, Jin J X, Li X D, Li Q L, Gu W D, et al. Single arc volumetric-modulated arc therapy is sufficient for nasopharyngeal carcinoma: a dosimetric comparison with dual arc VMAT and dynamic MLC and step-and-shoot intensity-modulated radiotherapy. Radiat Oncol, 2013; 8: 237-245.

12. Holt A, Van Gestel D, Arends M P, Korevaar E K, Schuring D,Kunze-Busch M C, et al. Multi-institutional comparison of volumetric modulated arc therapy vs. intensity-modulated radiation therapy for head-and-neck cancer: a planning study. Radiat Oncol, 2013; 8: 26-35.

13. Hansen C R, Bertelsen A, Hazell I, Zukauskaite R, Gyldenkerne N, Johansen J, et al. Automatic treatment planning improves the clinical quality of head and neck cancer treatment plans. Clin Transl Radiat Oncol, 2016; 1:2-8.

14. Hazell I, Bzdusek K, Kumar P, Hansen CR, Bertelsen A, Eriksen JG, et al. Automatic planning of head and neck treatment plans. J Appl Clin Med Phys, 2016; 17: 272-282.

15. Sharfo AW, Voet PW, Breedveld S, Mens JW, Hoogeman MS, Heijmen BJ. Comparison of VMAT and IMRT strategies for cervical cancer patients using automated planning. Radiother Oncol, 2015; 114: 395-401.

16. Mitchell R A, Wai P, Colgan R, Kieby AM, Donovan EM. Improving the efficiency of breast radiotherapy treatment planning using a semi-automated approach. J Appl Clin Med Phys, 2017; 18:18-24.

17. Tyagi N, Yang K, Gersten D, Yan D. A real time dose monitoring and dose reconstruction tool for patient specific VMAT QA and delivery. Med Phys 2012; 39:7194-204.

18. Webb S. Use of a quantitative index of beam modulation to characterize dose conformality: illustration by a comparison of full beamlet IMRT, few-segment IMRT (fsIMRT) and conformal unmodulated radiotherapy. Phys Med Biol, 2003; 48 : 2051-62.

19. Mcniven A L, Sharpe M B, Purdie T G. A new metric for assessing IMRT modulation complexity and plan deliverability. Med Phys, 2010; 37:505-515.

20. Dhanabalan R, Prakash J, Prabakar S, Ranganathan A, Johnjothi S, Nagarajan V. A study on the correlation between plan complexity and gamma index analysis in patient specific quality assurance of volumetric modulated arc therapy. Rep Pract Oncol Radiother, 2015; 20:57-65.

21. Wang Q, Dai J, Zhang K. A novel method for routine quality assurance of volumetric-modulated arc therapy. Med Phys, 2013; 40:1017121-10.

22. Lafond C, Chajon E, Devillers A, et al. Impact of MLC leaf width on volumetric-modulated arc therapy planning for head and neck cancers. J Appl Clin Med Phys, 2013:14:40-52.

23. Height F J, Kron T, Willis D, et al. Impact of MLC leaf width on the quality of the dose distribution in partial breast irradiation[J]. Med Dosi, 2012; 37:37-41.

24. Samuel B French, Stephen, et al. Adapting VMAT plans optimized for an HD120 MLC for delivery with a Millennium MLC. J Appl Clin Med Phys, 2017;18:143-151.

25. Qi P, Xia P. Relationship of segment area and monitor unit efficiency in aperture-based IMRT optimization. J Appl Clin Med Phys, 2013; 14:232-243.

26. Pogson E M, Aruguman S, Hansen C R, Currie M, Oborn BM, Blake SJ, et al. Multi-institutional comparison of simulated treatment delivery errors in ssIMRT, manually planned VMAT and autoplan-VMAT plans for nasopharyngeal radiotherapy. Phys Med, 2017; 42:55-66.

27. J. Osborn. Is VMAT beneficial for patients undergoing radiotherapy to the head and neck?. Radiography, 2016, 23(1):73.

28. Crowe S B, Kairn T, Kenny J, Knight RT, Hill B, Langton CM, et al. Treatment plan complexity metrics for predicting IMRT pre-treatment quality assurance results. Australas Phys Eng Sci Med, 2014; 37:475-482.

29. Li G, Wu K, Peng G, Zhang Y, Bai S. A retrospective analysis for patient-specific quality assurance of 
volumetric-modulated arc therapy plans. Med Dosi, 2015; 39: 309-313.

30. Mcgarry C K, Chinneck C D, O'Toole M M, O'Sullivan JM, Prise KM, Hounsell AR. Assessing software upgrades, plan properties and patient geometry using intensity modulated radiation therapy (IMRT) complexity metrics. Med Phys, 2011; 38: 2027-2034.

\section{Figures and tables}

DVH of breast

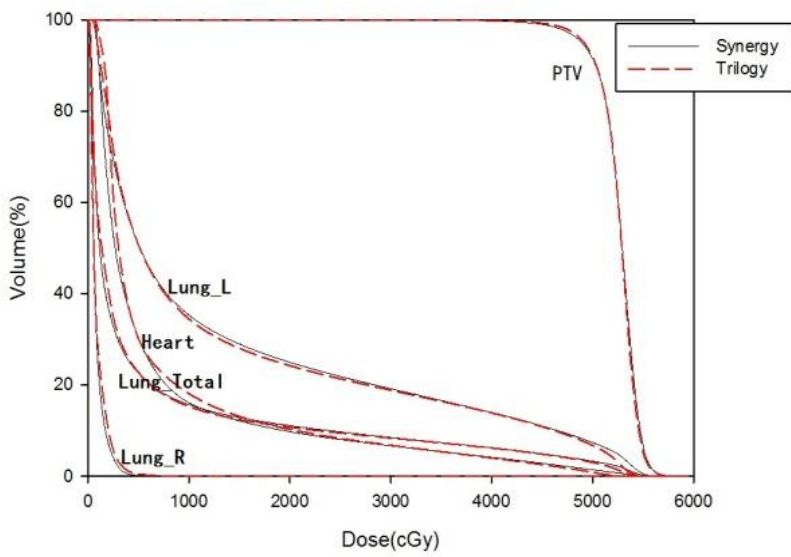

DVH of NPC

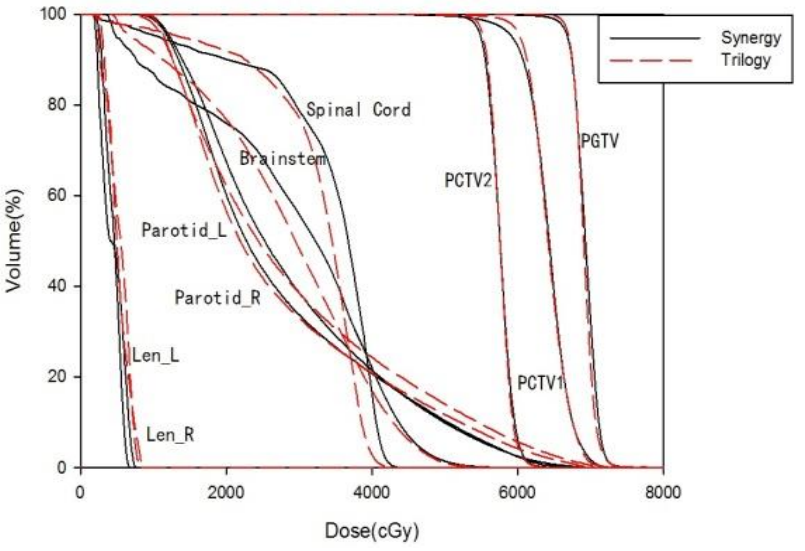

Fig. 1 Mean DVHs comparison between Trilogy and Synergy plans for BC(left) and NPC(right).

Solid line: Synergy; dashed line: Trilogy.

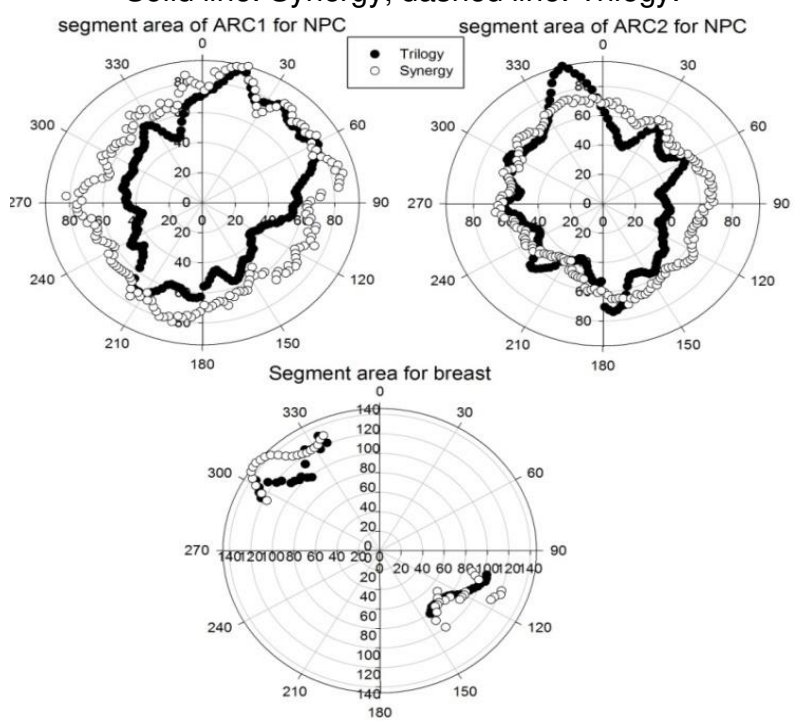

Fig. 2 Average SA comparison between Trilogy and Synergy plans for NPC and BC.

White circle: Synergy; black circle: Trilogy. 


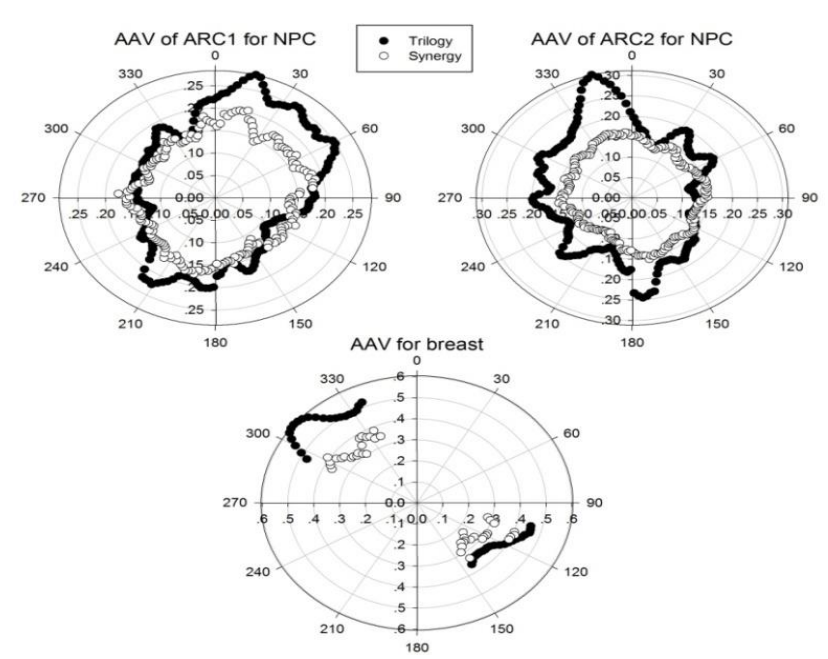

Fig. 3 Average AAV comparison between Trilogy and Synergy plans for NPC and BC. White circle: Synergy; black circle: Trilogy.

Table 1 Statistical summary of the differences in MU efficiency

\begin{tabular}{ccccc}
\hline & & \multicolumn{2}{c}{ MU } & P value \\
Site & Volume & Trilogy & Synergy & (Trilogy vs. Synergy) \\
\hline NPC & $833.4 \pm 174.8$ & $828.6 \pm 74.1$ & $782.9 \pm 85.2$ & 0.128 \\
BC & $833.3 \pm 198.9$ & $444.8 \pm 61.3$ & $393.8 \pm 75.3$ & 0.203 \\
P value & & & & \\
$(B C$ vs. NPC) & & 0.018 & 0.017 & \\
\hline
\end{tabular}

Table 2 Statistical summary of the differences in complexity scores

\begin{tabular}{ccccccc}
\hline \multirow{2}{*}{ Site } & Delivery & \multicolumn{5}{c}{ Complexity scores } \\
\cline { 2 - 6 } & System & SW $(\mathrm{cm})$ & SA $\left(\mathrm{cm}^{2}\right)$ & LSV & AAV & MCS \\
\hline \multirow{2}{*}{ NPC } & Trilogy & $6.87 \pm 1.95$ & $55.5 \pm 21.3$ & $0.84 \pm 0.033$ & $0.18 \pm 0.064$ & $0.14 \pm 0.016$ \\
& Synergy & $6.72 \pm 2.71$ & $66.3 \pm 17.9$ & $0.85 \pm 0.039$ & $0.14 \pm 0.037$ & $0.12 \pm 0.017$ \\
& P value & 0.000 & 0.000 & 0.000 & 0.000 & 0.002 \\
Breast & Trilogy & $8.84 \pm 2.56$ & $100.7 \pm 49.2$ & $0.89 \pm 0.026$ & $0.46 \pm 0.15$ & $0.42 \pm 0.106$ \\
& Synergy & $8.09 \pm 2.63$ & $108.5 \pm 42.7$ & $0.90 \pm 0.026$ & $0.33 \pm 0.13$ & $0.30 \pm 0.087$ \\
& P value & 0.000 & 0.000 & 0.031 & 0.000 & 0.001 \\
\hline
\end{tabular}

Table 3 Statistical summary of the differences in delivery efficiency and accuracy

\begin{tabular}{ccccccc}
\hline Site & Delivery & \multicolumn{5}{c}{ Delivery parameters } \\
\cline { 3 - 7 } & System & Gantry Speed & Dose Rate & \multicolumn{3}{c}{ Gamma Pass Rate } \\
\cline { 5 - 7 } & & $(\mathrm{deg} / \mathrm{s})$ & $(\mathrm{MU} / \mathrm{min})$ & $3 \mathrm{~mm} / 3 \%$ & $2 \mathrm{~mm} / 3 \%$ & $2 \mathrm{~mm} / 2 \%$ \\
\hline \multirow{2}{*}{ NPC } & Trilogy & $4.5 \pm 0.7$ & $242.1 \pm 166.7$ & $95.2 \pm 1.9$ & $90.3 \pm 3.3$ & $83.5 \pm 5.6$ \\
& Synergy & $4.1 \pm 0.8$ & $244.0 \pm 112.5$ & $93.3 \pm 0.9$ & $88.2 \pm 2.7$ & $77.3 \pm 2.2$ \\
Breast & Trilogy & $2.6 \pm 1.2$ & $531.8 \pm 144.4$ & $94.5 \pm 2.8$ & $88.5 .3 \pm 5.3$ & $81.0 \pm 6.4$ \\
& Synergy & $1.1 \pm 0.9$ & $258.7 \pm 192.9$ & $93.7 \pm 2.5$ & $87.9 \pm 3.9$ & $81.8 \pm 3.9$ \\
\hline
\end{tabular}


Figures
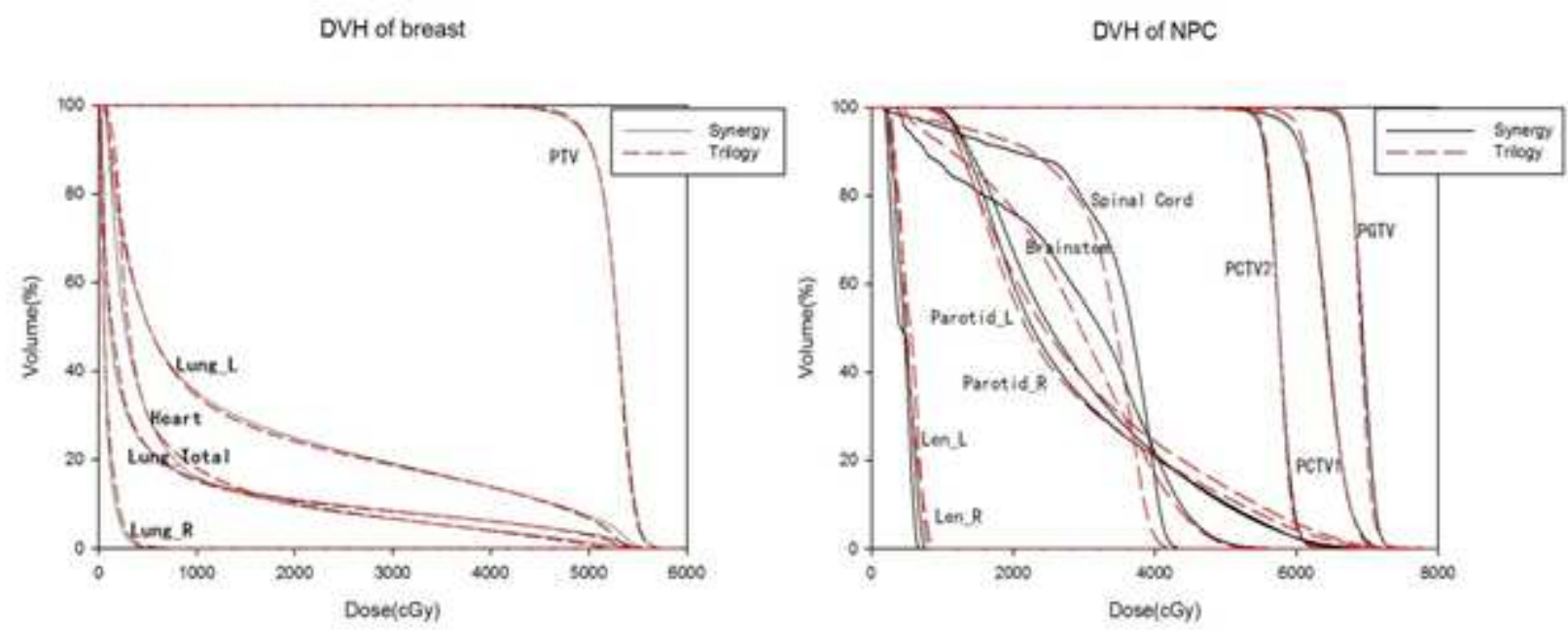

Figure 1

Mean DVHs comparison between Trilogy and Synergy plans for BC(left) and NPC(right). Solid line: Synergy; dashed line: Trilogy. 


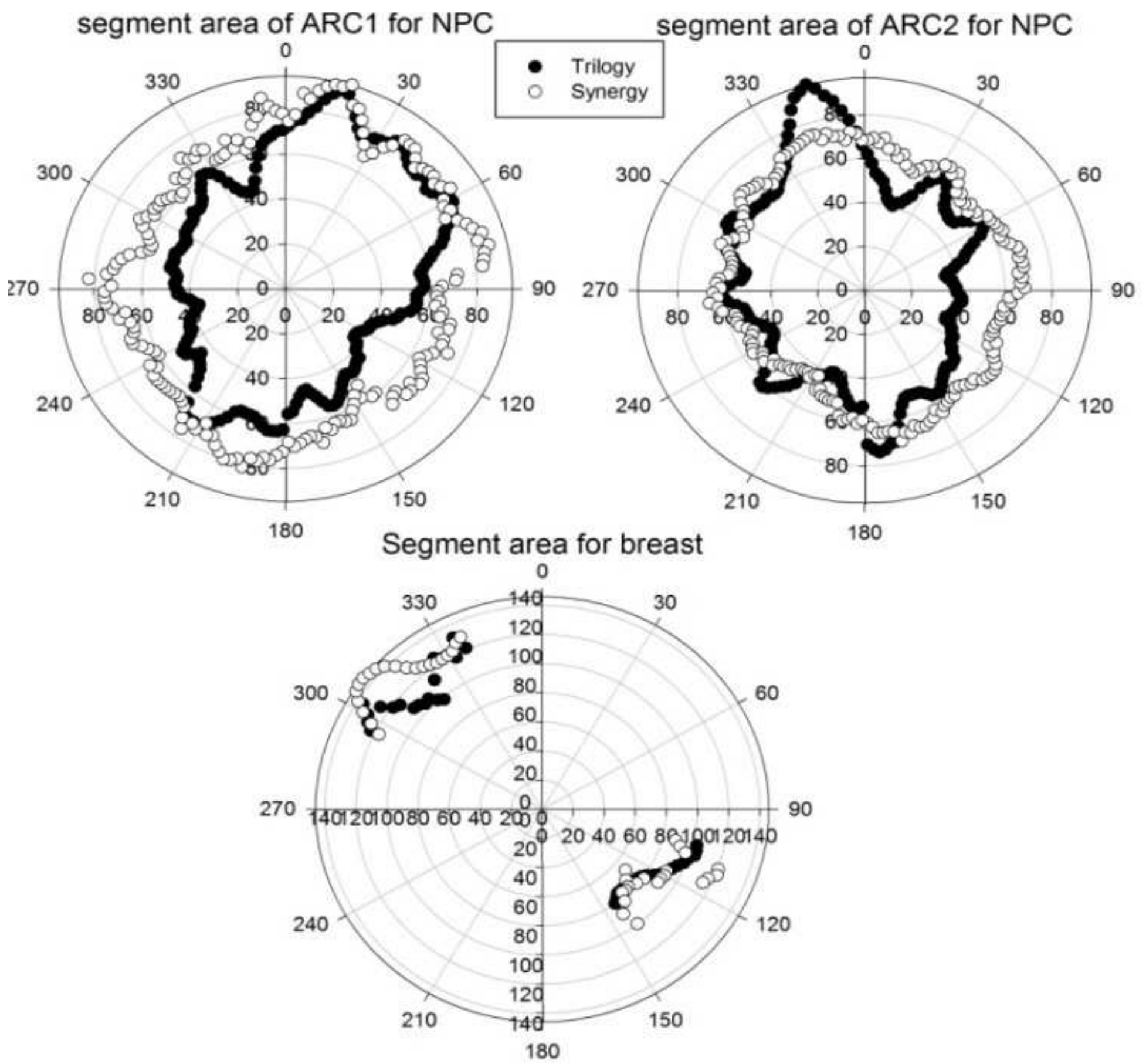

Figure 2

Average SA comparison between Trilogy and Synergy plans for NPC and BC. White circle: Synergy; black circle: Trilogy. 


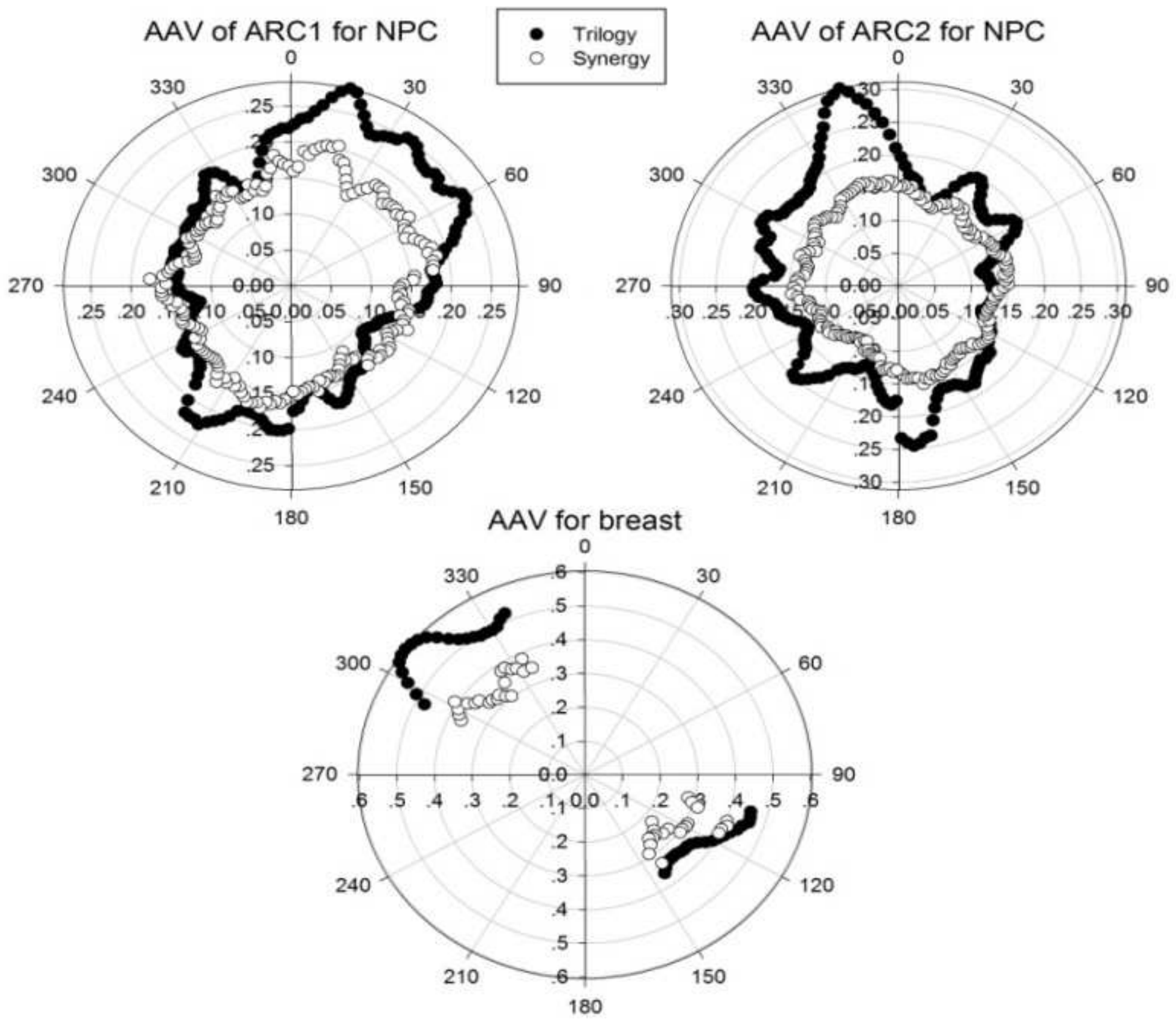

Figure 3

Average AAV comparison between Trilogy and Synergy plans for NPC and BC. White circle: Synergy; black circle: Trilogy. 